\title{
Updated Design of the CMB Polarization Experiment Satellite LiteBIRD
}

\author{
H. Sugai, et al. [full author details at the end of the article]
}

Received: 30 August 2019 / Accepted: 26 December 2019 / Published online: 27 January 2020

(c) The Author(s) 2020

\begin{abstract}
Recent developments of transition-edge sensors (TESs), based on extensive experience in ground-based experiments, have been making the sensor techniques mature enough for their application on future satellite cosmic microwave background (CMB) polarization experiments. LiteBIRD is in the most advanced phase among such future satellites, targeting its launch in Japanese Fiscal Year 2027 (2027FY) with JAXA's H3 rocket. It will accommodate more than 4000 TESs in focal planes of reflective low-frequency and refractive medium-and-high-frequency telescopes in order to detect a signature imprinted on the $\mathrm{CMB}$ by the primordial gravitational waves predicted in cosmic inflation. The total wide frequency coverage between 34 and $448 \mathrm{GHz}$ enables us to extract such weak spiral polarization patterns through the precise subtraction of our Galaxy's foreground emission by using spectral differences among $\mathrm{CMB}$ and foreground signals. Telescopes are cooled down to $5 \mathrm{~K}$ for suppressing thermal noise and contain polarization modulators with transmissive half-wave plates at individual apertures for separating sky polarization signals from artificial polarization and for mitigating from instrumental $1 / f$ noise. Passive cooling by using V-grooves supports active cooling with mechanical coolers as well as adiabatic demagnetization refrigerators. Sky observations from the second SunEarth Lagrangian point, L2, are planned for 3 years. An international collaboration between Japan, the USA, Canada, and Europe is sharing various roles. In May 2019, the Institute of Space and Astronautical Science, JAXA, selected LiteBIRD as the strategic large mission No. 2.
\end{abstract}

Keywords Satellite $\cdot$ Cosmic microwave background $\cdot$ Polarization · Inflation · Primordial gravitational wave

\section{Concept of LiteBIRD}

It has been suggested since the 1980s [1-3] that inflation occurred in the very early, high energy Universe, to resolve remaining issues of the Big Bang theory, such as uniformity, flatness, and monopole problems. Cosmic inflation predicts primordial gravitational wave production, with quantum fluctuations of spacetime 

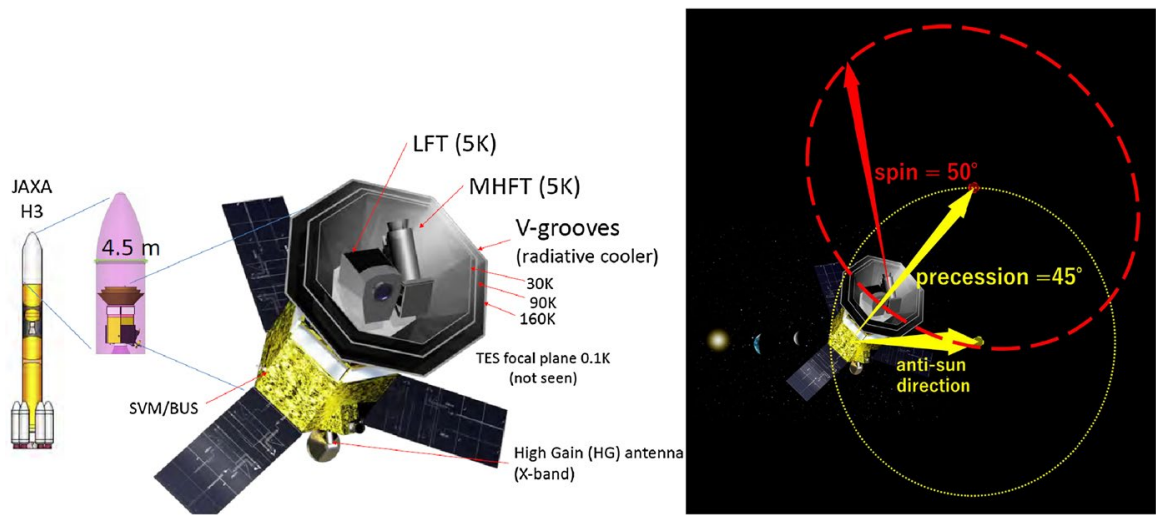

Fig. 1 Left: Conceptual design of LiteBIRD. Warm launch is planned with JAXA's H3 rocket. Right: Scans with spin angle $50^{\circ}$ and precession angle $45^{\circ}$ at L2. (Color figure online)

as its origin. The LiteBIRD (Lite (Light) satellite for the studies of B-mode polarization and Inflation from cosmic background Radiation Detection) aims to detect signatures of these primordial gravitational waves in the form of specific curl patterns $[4,5]$ of polarization angle distribution of the Cosmic Microwave Background $(\mathrm{CMB})$. The sizes of expected spiral patterns in the sky are characterized by Hubble lengths at the electron scattering eras of the $\mathrm{CMB}$, since primordial gravitational waves that enter the horizon in these eras most effectively produce tensor anisotropies. It is therefore essential to cover the whole sky to investigate these large Hubble lengths at the recombination era as well as the reionization era.

LiteBIRD is focused on this point: targeting [6] both the recombination era with the multipole moment 1 between 11 and 200 and the reionization era with 1 between 2 and 10, optimizing the angular resolution. The other important concepts of this satellite are a warm launch without the requirements of heavy vessels/tanks and use of multichroic detectors for the effective exploitation of finite focal-plane areas. Advantages of measurements from space are being free from atmospheric effects, providing high sensitivity, stability with less systematic uncertainties [e.g., 7], and no restrictions on observing band selection. Space measurements also give no pickup from the ground. The Sun-Earth L2 point has been selected, since the Sun, the Earth, and the Moon are all located in almost the same direction, which makes it easier to avoid facing them in terms of optical and thermal aspects. Care should be taken, however, on cosmic ray effects [8] because the satellite is more directly exposed to them. Sky observations are planned for 3 years: The presently guaranteed cooling-chain lifetime is 3.5 years, in which 0.5 year is assigned to the transitional period to the normal observation phase on course to L2. A scanning strategy with a combination of boresight spin angle of $50^{\circ}$ around the satellite axis and its precession-like rotation around the anti-Sun direction of $45^{\circ}$ is used (Fig. 1), since this combination provides not only a fairly 
uniform sky coverage but also the minimization of instrumental systematic uncertainties in polarization measurements.

\section{Status of LiteBIRD}

In May 2019, the Institute of Space and Astronautical Science (ISAS), JAXA, confirmed that LiteBIRD completed activities planned during Pre-phase A2 (previously called Phase-A1 and dedicated to the concept study and development of key technologies) and selected LiteBIRD as the strategic large mission No. 2, with modifications to the cooling chain and subsequently the focal-plane designs: We have chosen to use adiabatic demagnetization refrigerators (ADRs) in series, removing ideas $[6,9]$ of using a JAXA-provided 1-K J-T cooler and of having $2-\mathrm{K}$ telescope apertures. To recover the degraded sensitivity with $5-\mathrm{K}$ apertures

Table 1 Updated basic parameters and current baseline design for LiteBIRD

\begin{tabular}{ll}
\hline Mission category & JAXA's strategic large mission \\
Launch vehicle & H3-22L or equivalent \\
Launch schedule & $2027 \mathrm{FY}$ \\
Ground station & JAXA's ground stations (USC, GREAT) \\
Observation period & 3 years \\
Uncertainty of tensor-to- & $\delta r<1 \times 10^{-3}$ \\
scalar ratio $r$ & $2 \leqq 1 \leqq 200$ \\
Multipole moment & Second Sun-Earth Lagrangian point L2; Lissajous orbit \\
Orbit & Precession angle 45 (10 ${ }^{-2}-10^{-3}$ rpm); spin angle 50 (0.05-0.1 rpm) \\
Scan & $<2.1$ arcmin \\
Pointing knowledge & Radiative cooling and mechanical refrigerators (Stirling and JT) without \\
Cooling system & cryogens. Cool in space after launch. ADRs are used to cool the focal plane \\
& down to $100 \mathrm{mK}$ \\
Focal-plane detector & bultichroic superconducting detector arrays with more than 4000 TES \\
Sensitivity & $2 \mu \mathrm{K}$ arcmin \\
Observing frequencies & 15 bands between 34 and 448 GHz \\
Modulation & Satellite spin and half-wave plate modulation \\
Data transfer & 9.6 GByte/day \\
Mass & 2.6 ton \\
Electrical power & $3.0 \mathrm{~kW}$ \\
\hline
\end{tabular}


Table 2 LiteBIRD telescope parameters

\begin{tabular}{llll}
\hline Telescope & Low freq. & Medium freq. & High freq. \\
\hline Frequency & $34-161 \mathrm{GHz}$ & $89-224 \mathrm{GHz}$ & $166-448 \mathrm{GHz}$ \\
Telescope field of view & $20^{\circ} \times 10^{\circ}$ & $28^{\circ}$ diameter & $28^{\circ}$ diameter \\
Aperture diameter & $400 \mathrm{~mm}$ & $300 \mathrm{~mm}$ & $200 \mathrm{~mm}$ \\
Angular resolution & $70-24$ arcmin & $38-28$ arcmin & $29-18$ arcmin \\
Rotational HWP & $46-83 \mathrm{rpm}$ & $39-70 \mathrm{rpm}$ & $61-110 \mathrm{rpm}$ \\
Number of detectors & 1248 & 2074 & 1354 \\
\hline
\end{tabular}

instead, we have increased the number of detectors. With the international collaboration between Japan, the USA, Canada, and Europe, the satellite is planned to be launched with JAXA's H3 rocket in 2027FY (Fig. 1). Tables 1 and 2 summarize the updated basic parameters and current baseline design.

\section{Current Design and Technical Progresses of LiteBIRD}

\subsection{Cooling Chain}

With the design modification required, we have selected the following baseline combination for the cooling chain: (i) down to $5 \mathrm{~K}$ a sunshield and passive cooling with V-grooves [10], 15-K pulse tube coolers, and a 4-K J-T with 2ST precoolers; (ii) from 5 to $1.75 \mathrm{~K}$ a parallel three-stage ADR for providing continuous cooling at $1.75 \mathrm{~K}$; and (iii) from $1.75 \mathrm{~K}$ to $100 \mathrm{mK}$ a multistaged ADR with continuous cooling at $300 \mathrm{mK}$ and $100 \mathrm{mK}$. Detailed schemes for this cooling chain are described in [11].

\subsection{Telescopes and Polarization Modulator Units}

The accurate, precise, and robust foreground cleaning requires 15 frequency bands covering a wide range of 34 to $448 \mathrm{GHz}$. This will be achieved with two kinds of telescopes (Table 2): a reflective one for the lower-frequency range (LFT) and refractive ones for medium- and high-frequency ranges (MHFT [12]). While the LFT uses crossed-Dragone reflective optics $[13,14]$ to minimize effects from multireflection among refractive surfaces, the MHFT uses lens optics with the advantage of compactness. For the LFT, the F\#3.0 and the crossing angle of $90^{\circ}$ have been selected, since this combination suppresses the straylight [9, 15] (Fig. 2), and aluminum is used as the mirror material [16]. All the telescopes are cooled down to $5 \mathrm{~K}$ for suppressing thermal noise.

Each telescope has a polarization modulator unit (PMU) [12, 17-19], which consists of a transmissive half-wave plate (HWP) system rotated with a superconductive magnetic bearing (SMB), as the first optical element, close to the aperture stop 

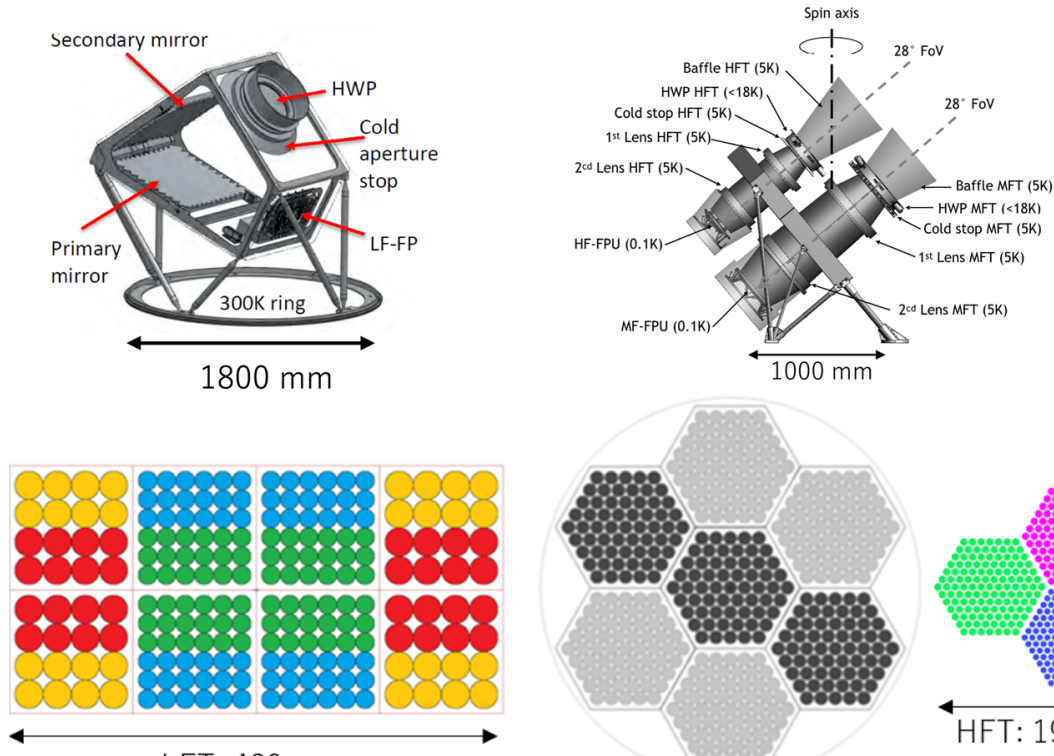

LFT: $420 \mathrm{~mm}$
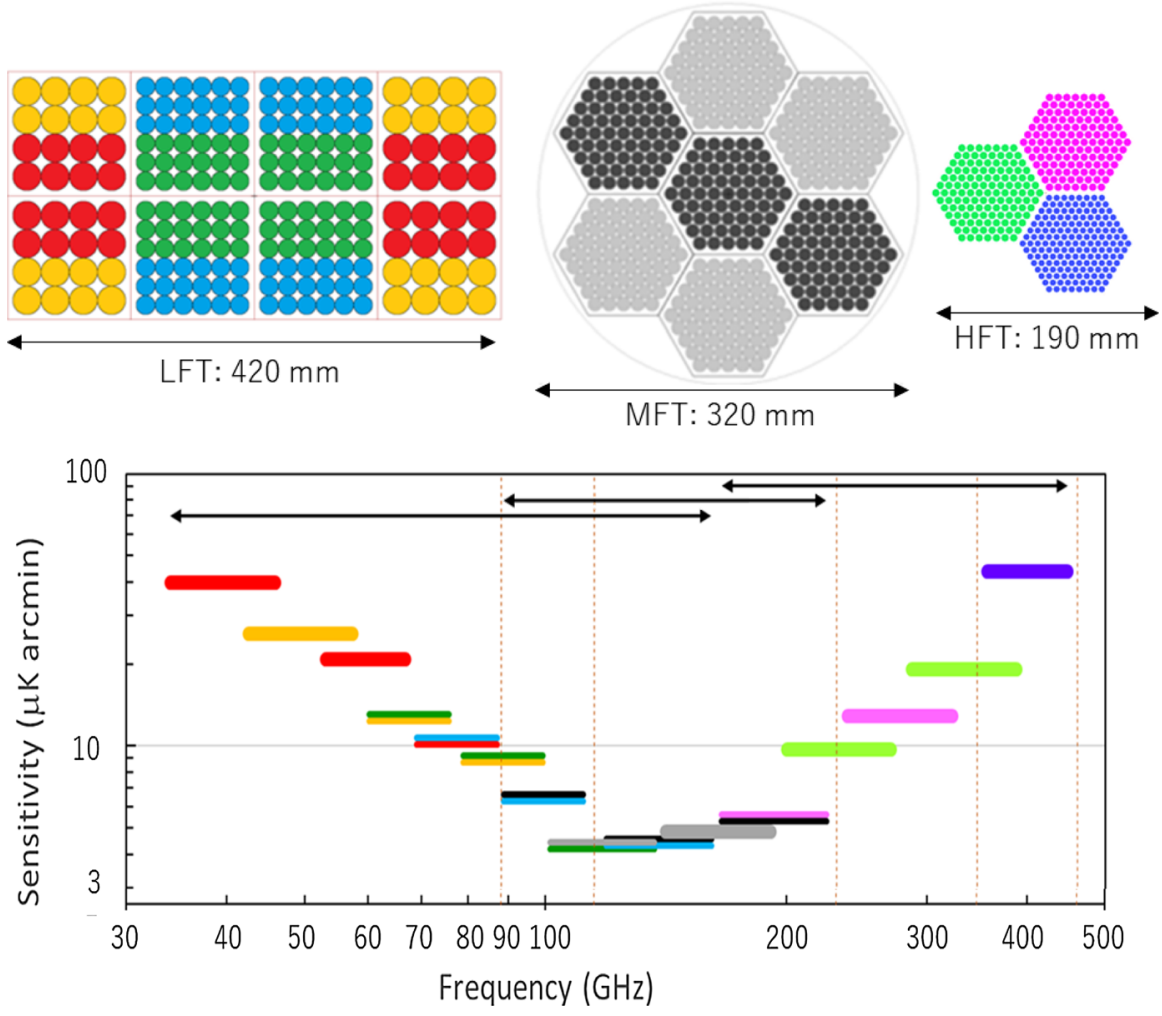

Fig. 2 Top: Designs of LFT (left) [9] and MHFT (right) [12]. Middle: Pixel distribution in LFT (left), MFT (center), and HFT (right) focal planes. Pixel diameters are: $23.6 \mathrm{~mm}$ for red (central frequencies of $40,60,78 \mathrm{GHz})$ and orange $(50,68,89 \mathrm{GHz})$ pixels; $15.6 \mathrm{~mm}$ for green $(68,89,119 \mathrm{GHz})$ and light blue $(78,100,140 \mathrm{GHz})$ pixels in LFT; $11.6 \mathrm{~mm}$ for black $(100,140,195 \mathrm{GHz})$ and gray $(119,166 \mathrm{GHz})$ pixels in MFT; $6.6 \mathrm{~mm}$ for pink $(195,280 \mathrm{GHz})$ and light green $(235,337 \mathrm{GHz})$ pixels; and $5.7 \mathrm{~mm}$ for blue $(402 \mathrm{GHz})$ pixels in HFT. Bottom: Predicted LiteBIRD sensitivity. Colors correspond to those shown in the pixel distributions. Frequency ranges for LFT, MFT, and HFT are shown with black arrows. Frequencies of CO $J=1-0,2-1,3-2,4-3$ and HCN $J=1-0$ lines are shown as thin vertical brown dashed lines. Total sensitivity is $2 \mu \mathrm{K}$ arcmin. (Color figure online)

to distinguish the sky and instrumental polarization components and to reduce the instrumental $1 / f$ noise. The SMB enables contactless operation of the HWP system, which minimizes heat generation, through magnetic levitation by using YBCO bulk 
and permanent SmCo magnets. The LFT HWP system consists of multilayer stacked sapphire HWPs with optical axes shifted relative to each other to achieve high polarization efficiency over the wide frequency range [20], while the MHFT employs the metal-mesh HWPs [19]. At the top and bottom surfaces of the LFT HWP system, we adopt anti-reflection with sub-wavelength structure produced by laser machining. A cryogenic testbed is designed to study interactions, such as between spaceoptimized detectors and the PMU, consisting of a magnetically levitated and rotating HWP system [21].

\subsection{Detectors and Readout}

Transition-edge sensor (TES) bolometers are used to form multichroic pixels, based on maturity from successful ground-based and balloon experiments [22]. TES bolometers are coupled with a silicon lenslet and a sinuous antenna for individual broadband pixels for LFT and MFT, while silicon platelet-based corrugated horn and orthomode transducers are used for HFT [23, 24]. The noise equivalent power of TES bolometer is proportional to $\sqrt{ }\left(P_{\text {sat }} T_{\mathrm{b}}\right)$, with the $T_{\mathrm{c}} / T_{\mathrm{b}}$ ratio being optimized. Here, $P_{\text {sat }}, T_{\mathrm{c}}$, and $T_{\mathrm{b}}$ are the saturated power, transition temperature, and thermal bath temperature, respectively. The TES parameter optimization for LiteBIRD, toward low-saturation power detectors for the satellite environment, has been carried out, as well as a sensor impedance that readily couples to the frequency-domain multiplexer. The electro-thermal time constants are controlled by slowing down with the additional heat capacity. Details of these experimental results are described in [25]. Cosmic ray mitigation methods have been developed by reducing propagation with palladium structures to absorb phonons and with the removal of bulk silicon to block phonons [26]. Readout will be carried out through digital frequency multiplexing, with 68 bolometers connected to each SQUID array amplifier [27, 28]. The updated focal-plane designs and the expected sensitivities are shown in Fig. 2.

\section{Foreground-Cleaning and Systematic Uncertainty Studies}

The focal-plane designs have been determined through iterations with sensitivity calculations based on thermal studies [10], as well as with foreground-cleaning (by using methods described in [29-32]) and systematic uncertainty studies. The baseline foreground-cleaning results have been obtained based on an eight-dimensional parameterization of the foregrounds, including synchrotron power-law spectral index plus curvature and effective temperature of dust, plus power-law index of dust emissivity for $Q$ and $U$ Stokes parameters, each of which is allowed to vary across the sky. Systematic uncertainties have also been studied [33], including beam systematics [14], instrumental polarization and HWP harmonics, polarization efficiency, relative and absolute gain, pointing, polarization angle, time-correlated noise, cosmic ray glitches, bandpass mismatch [34], transfer function, nonlinearity, and non-uniformity in HWP, with realistic ground/in-flight calibration methods taken into account [e.g., 15, 35]. Through all these studies, it has been shown that the 
updated focal-plane designs satisfy LiteBIRD's full success of the total uncertainty in the tensor-to-scalar ratio of less than 0.001 , with its uncertainty budgets distributed comparably into a statistical part (including foreground residual and lensing B-mode), a systematic part, and a margin.

Acknowledgements This work was supported by World Premier International Research Center Initiative (WPI), MEXT, Japan, by JSPS Core-to-Core Program, A. Advanced Research Networks, and by JSPS KAKENHI Grant Numbers JP15H05891, JP17H01115, and JP17H01125. The Italian contribution to the LiteBIRD phase A is supported by the Italian Space Agency (ASI Grant No. 2016-24- H.12018) and the National Institute for Nuclear Physics (INFN). The French contribution to the LiteBIRD phase A is supported by the Centre National d'Etudes Spatiale (CNES), by the Centre National de la Recherche Scientifique (CNRS), and by the Commissariat à l'Energie Atomique (CEA). A Concurrent Design Facility study focused on the MHFT and Sub-Kelvin coolers has been led by the European Space Agency (ESA). The Canadian contribution to LiteBIRD is supported by the Canadian Space Agency. The US contribution is supported by NASA Grant no. 80NSSC18K0132.

Open Access This article is licensed under a Creative Commons Attribution 4.0 International License, which permits use, sharing, adaptation, distribution and reproduction in any medium or format, as long as you give appropriate credit to the original author(s) and the source, provide a link to the Creative Commons licence, and indicate if changes were made. The images or other third party material in this article are included in the article's Creative Commons licence, unless indicated otherwise in a credit line to the material. If material is not included in the article's Creative Commons licence and your intended use is not permitted by statutory regulation or exceeds the permitted use, you will need to obtain permission directly from the copyright holder. To view a copy of this licence, visit http://creativecommons.org/licen ses/by/4.0/.

\section{References}

1. A.A. Starobinsky, Phys. Lett. 91B, 99-102 (1980)

2. K. Sato, Mon. Notices. R. Astron. Soc. 195, 467-479 (1981)

3. A.H. Guth, Phys. Rev. D 23, 347-356 (1981)

4. U. Seljak, M. Zaldarriaga, Phys. Rev. Lett. 78, 2054-2057 (1997)

5. M. Kamionkowski, A. Kosowsky, A. Stebbins, Phys. Rev. Lett. 78, 2058-2061 (1997)

6. M. Hazumi et al., J. Low Temp. Phys. 194, 443-452 (2019). https://doi.org/10.1007/s10909-01902150-5

7. S. Takakura et al., Astrophys. J. 870, 102 (2019). https://doi.org/10.3847/1538-4357/aaf381

8. A. Miniussi et al., J. Low Temp. Phys. 176, 815-821 (2014). https://doi.org/10.1007/s1090 9-014-1104-X

9. Y. Sekimoto et al., SPIE Proc. 10698, 106981Y (2018). https://doi.org/10.1117/12.2313432

10. T. Hasebe et al., SPIE Proc. 10698, 1069864 (2018). https://doi.org/10.1117/12.2313034

11. J.-M. Duval, et al., J. Low Temp. Phys. This Special Issue (2020) (in press)

12. B. Mot, et al., J. Low Temp. Phys. This Special Issue (2020) (in press)

13. S. Kashima, M. Hazumi, H. Imada, N. Katayama, T. Matsumura, Y. Sekimoto, H. Sugai, Appl. Opt. 57, 4171-4179 (2018). https://doi.org/10.1364/AO.57.004171

14. H. Imada et al., SPIE Proc. 10698, 106984K (2018). https://doi.org/10.1117/12.2312185

15. H. Takakura et al., IEEE Trans. Terahertz Sci. Technol. 9, 598 (2019). https://doi.org/10.1109/ TTHZ.2019.2937497

16. H. Sugai et al., SPIE Proc. 10372, 103720I (2017). https://doi.org/10.1117/12.2273765

17. Y. Sakurai et al., SPIE Proc. 10708, 107080E (2018). https://doi.org/10.1117/12.2312391

18. F. Columbro, P. de Bernardis, S. Masi, Rev. Sci. Instrum. 89, 125004 (2018). https://doi. org/10.1063/1.5035332

19. G. Pisano et al., SPIE Proc. 9153, 915317 (2014). https://doi.org/10.1117/12.2056380

20. K. Komatsu et al., SPIE Proc. 10708, 1070847 (2018). https://doi.org/10.1117/12.2312431 
21. T. Ghigna, et al., J. Low Temp. Phys. This Special Issue (2020). https://doi.org/10.1007/s1090 9-020-02359-9

22. A. Suzuki, et al., J. Low Temp. Phys. This Special Issue (2020). https://doi.org/10.1007/s1090 9-019-02325-0

23. B. Westbrook, et al., J. Low Temp. Phys. This Special Issue (2020) (in press)

24. A. Suzuki et al., J. Low Temp. Phys. 193, 1048-1056 (2018). https://doi.org/10.1007/s1090 9-018-1947-7

25. G. Jaehnig, et al., J. Low Temp. Phys. This Special Issue (2020) (in press)

26. Y. Minami, et al., J. Low Temp. Phys. This Special Issue (2020) (in press)

27. M. Tsujimoto et al., SPIE Proc. 10698, 1069847 (2018). https://doi.org/10.1117/12.2311579

28. A. Lowitz, A. Bender, M.A. Dobbs, A. Gilbert, SPIE Proc. 10708, 107081D (2018). https://doi. org/10.1117/12.2311984

29. J. Errard, R. Stompor, arXiv:1811.00479v1 (2018)

30. M. Remazeilles, C. Dickinson, H.K.K. Eriksen, I.K. Wehus, Mon. Notices. R. Astron. Soc. 458, 2032-2050 (2016). https://doi.org/10.1093/mnras/stw441

31. H.K. Eriksen, J.B. Jewell, C. Dickinson, A.J. Banday, K.M. Górski, C.R. Lawrence, Astrophys. J. 676, 10-32 (2008). https://doi.org/10.1086/525277

32. K. Ichiki, H. Kanai, N. Katayama, E. Komatsu, Prog. Theor. Exp. Phys. 2019, 033E01 (2019). https://doi.org/10.1093/ptep/ptz009

33. H. Ishino et al., SPIE Proc. 9904, 99040X (2016). https://doi.org/10.1117/12.2231995

34. D.-T. Hoang et al., J. Cosmol. Astropart. Phys. 2017, 015 (2017). https://doi. org/10.1088/1475-7516/2017/12/015

35. Y. Minami, H. Ochi, K. Ichiki, N. Katayama, E. Komatsu, T. Matsumura arXiv: 1904.12440v1 (2019)

Publisher's Note Springer Nature remains neutral with regard to jurisdictional claims in published maps and institutional affiliations.

\section{Affiliations}

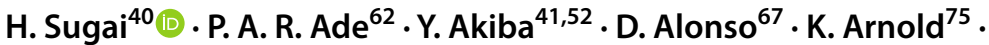
J. Aumont ${ }^{11}$. J. Austermann ${ }^{72}$. C. Baccigalupi ${ }^{19,25,27}$. A. J. Banday ${ }^{11}$. R. Banerji ${ }^{57}$ - R. B. Barreiro ${ }^{59} \cdot$ S. Basak ${ }^{16,27}$. J. Beall ${ }^{72} \cdot$ S. Beckman ${ }^{74}$. M. Bersanelli2,30 $\cdot$ J. Borrill ${ }^{70,74} \cdot$ F. Boulanger ${ }^{9}$ M. L. Brown ${ }^{66} \cdot$ M. Bucher $^{5}$. A. Buzzelli ${ }^{29} \cdot$ E. Calabrese ${ }^{62} \cdot$ F. J. Casas ${ }^{59} \cdot$ A. Challinor ${ }^{63,65} \cdot$ V. Chan ${ }^{4}$. Y. Chinone ${ }^{40,74}$ - J.-F. Cliche ${ }^{1}$ - F. Columbro ${ }^{24,26}$ - A. Cukierman ${ }^{74}$.

D. Curtis ${ }^{74}$ - P. Danto . P. de Bernardis ${ }^{24,26}$ - T. de Haan ${ }^{70}$ - M. De Petris ${ }^{24,26}$.

C. Dickinson ${ }^{66} \cdot$ M. Dobbs ${ }^{1} \cdot$ T. Dotani ${ }^{37} \cdot$ L. Duband ${ }^{6} \cdot$ A. Ducout ${ }^{40}$.

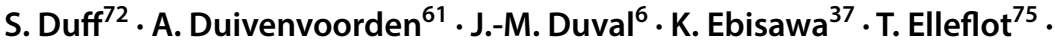

H. Enokida ${ }^{35} \cdot$ H. K. Eriksen ${ }^{57}$. J. Errard ${ }^{5} \cdot$ T. Essinger-Hileman ${ }^{71} \cdot$ F. Finelli ${ }^{20}$.

R. Flauger ${ }^{75}$. C. Franceschet ${ }^{22,30} \cdot$ U. Fuskeland ${ }^{57} \cdot$ K. Ganga $^{5} \cdot$ J.-R. Gao G $^{56}$.

R. Génova-Santos ${ }^{58,60} \cdot$ T. Ghigna ${ }^{40,67}$ - A. Gomez ${ }^{7}$ - M. L. Gradziel ${ }^{17}$.

J. Grain ${ }^{9}$ - F. Grupp ${ }^{14,15}$ - A. Gruppuso ${ }^{20}$ - J. E. Gudmundsson ${ }^{61}$.

N. W. Halverson ${ }^{76} \cdot$ P. Hargrave ${ }^{62} \cdot$ T. Hasebe $^{37} \cdot$ M. Hasegawa ${ }^{41,52}$.

M. Hattori ${ }^{53} \cdot$ M. Hazumi ${ }^{37,40,41,52} \cdot$ S. Henrot-Versille ${ }^{12} \cdot$ D. Herranz ${ }^{59}$.

C. Hill ${ }^{70,74}$ - G. Hilton ${ }^{72}$ - Y. Hirota ${ }^{35}$ - E. Hivon ${ }^{8} \cdot$ R. Hlozek ${ }^{4}$ - D.-T. Hoang ${ }^{68,77}$. J. Hubmayr ${ }^{72} \cdot$ K. Ichiki ${ }^{45} \cdot$ T. Iida ${ }^{40} \cdot$ H. Imada ${ }^{12} \cdot$ K. Ishimura ${ }^{54} \cdot$ H. Ishino ${ }^{41,48}$. G. C. Jaehnig ${ }^{76} \cdot$ M. Jones ${ }^{67} \cdot$ T. Kaga ${ }^{37}$ - S. Kashima ${ }^{46}$. Y. Kataoka ${ }^{48}$. 


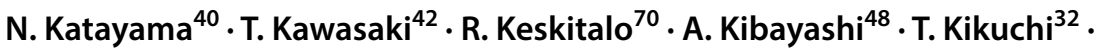

K. Kimura ${ }^{49} \cdot$ T. Kisner ${ }^{70}$-Y. Kobayashi ${ }^{38} \cdot$ N. Kogiso ${ }^{49} \cdot$ A. Kogut $^{71} \cdot$ K. Kohri $^{41}$.

E. Komatsu ${ }^{13} \cdot$ K. Komatsu ${ }^{48} \cdot$ K. Konishi ${ }^{36} \cdot$ N. Krachmalnicoff ${ }^{19,25,27}$.

C. L. Kuo ${ }^{69,73} \cdot$ N. Kurinsky ${ }^{69,73} \cdot$ A. Kushino ${ }^{44} \cdot$ M. Kuwata-Gonokami ${ }^{34}$.

L. Lamagna ${ }^{24,26} \cdot$ M. Lattanzi ${ }^{21}$ - A. T. Lee ${ }^{70,74}$ - E. Linder ${ }^{70,74} \cdot$ B. Maffei ${ }^{9}$.

D. Maino ${ }^{22,30} \cdot$ M. Maki ${ }^{41}$ - A. Mangilli ${ }^{11}$ - E. Martínez-González ${ }^{59} \cdot$ S. Masi ${ }^{24,26}$.

R. Mathon ${ }^{11} \cdot$ T. Matsumura ${ }^{40} \cdot$ A. Mennella ${ }^{22,30} \cdot$ M. Migliaccio ${ }^{24,29}$.

Y. Minami ${ }^{41} \cdot$ K. Mistuda ${ }^{37} \cdot$ D. Molinari ${ }^{28} \cdot$ L. Montier $^{11} \cdot$ G. Morgante ${ }^{20}$.

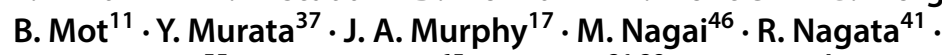

S. Nakamura ${ }^{55} \cdot$ T. Namikawa ${ }^{65} \cdot$ P. Natoli ${ }^{21,28} \cdot$ S. Nerval ${ }^{4}$. T. Nishibori ${ }^{39}$.

H. Nishino ${ }^{50} \cdot$ Y. Nomura ${ }^{51} \cdot$ F. Noviello ${ }^{62} \cdot$ C. O'Sullivan $^{17} \cdot$ H. Ochi $^{55}$.

H. Ogawa ${ }^{49} \cdot$ H. Ogawa ${ }^{37} \cdot$ H. Ohsaki ${ }^{35} \cdot$ I. Ohta $^{43} \cdot$ N. Okada ${ }^{37} \cdot$ N. Okada ${ }^{49}$.

L. Pagano ${ }^{21,28} \cdot$ A. Paiella $24,26 \cdot$ D. Paoletti ${ }^{20} \cdot$ G. Patanchon $^{5} \cdot$ F. Piacentini ${ }^{24,26}$.

G. Pisano ${ }^{62} \cdot$ G. Polenta ${ }^{18} \cdot$ D. Poletti ${ }^{19,25,27} \cdot$ T. Prouvé $^{6} \cdot$ G. Puglisi ${ }^{73}$.

D. Rambaud ${ }^{11}$. C. Raum ${ }^{74}$. S. Realini ${ }^{22,30} \cdot$ M. Remazeilles $^{66} \cdot$ G. Roudil ${ }^{11}$.

J. A. Rubiño-Martín ${ }^{58,60} \cdot$ M. Russell ${ }^{75} \cdot$ H. Sakurai ${ }^{34} \cdot$ Y. Sakurai $^{40} \cdot$ M. Sandri ${ }^{20}$.

G. Savini ${ }^{64}$ - D. Scott ${ }^{3}$. Y. Sekimoto ${ }^{37,41,52}$ - B. D. Sherwin ${ }^{63,65} \cdot$ K. Shinozaki ${ }^{39}$.

M. Shiraishi ${ }^{47}$ - P. Shirron ${ }^{71}$ - G. Signorelli ${ }^{23}$ - G. Smecher ${ }^{2} \cdot$ P. Spizzi ${ }^{7}$.

S. L. Stever ${ }^{40} \cdot$ R. Stompor ${ }^{5} \cdot$ S. Sugiyama ${ }^{51} \cdot$ A. Suzuki $^{70} \cdot$ J. Suzuki ${ }^{41}$.

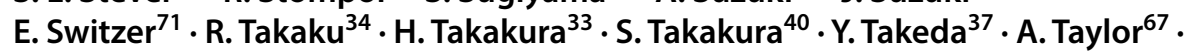

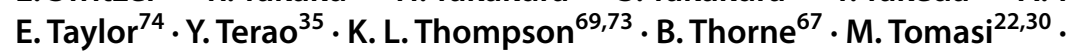

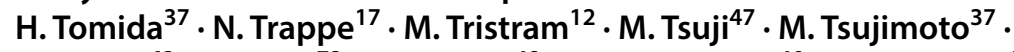

C. Tucker ${ }^{62} \cdot$ J. Ullom $^{72} \cdot$ S. Uozumi ${ }^{48} \cdot$ S. Utsunomiya ${ }^{40} \cdot$ J. Van Lanen $^{72}$.

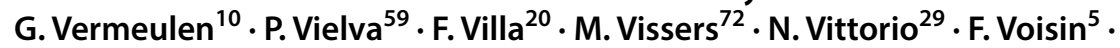

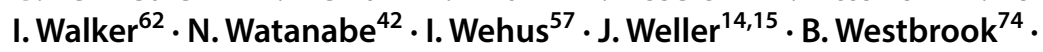

B. Winter ${ }^{64} \cdot$ E. Wollack ${ }^{71} \cdot$ R. Yamamoto ${ }^{32} \cdot$ N. Y. Yamasaki ${ }^{37} \cdot$ M. Yanagisawa $^{48}$.

T. Yoshida ${ }^{37} \cdot$ J. Yumoto ${ }^{34} \cdot$ M. Zannoni ${ }^{22,31} \cdot$ A. Zonca $^{75}$

H. Sugai

hajime.sugai@ipmu.jp

1 McGill University, Montreal, Canada

2 Three-Speed Logic, Inc., Victoria, Canada

3 University of British Columbia, Vancouver, Canada

4 University of Toronto, Toronto, Canada

5 APC, CNRS, University Paris Diderot, Paris, France

6 Univ. Grenoble Alpes, CEA, Grenoble, France

7 CNES, Paris, France

8 IAP, Sorbonne University, Paris, France

9 IAS, Université Paris-Saclay, CNRS, Orsay, France

10 Institut Néel, Grenoble, France

11 IRAP, University de Toulouse, CNRS, CNES, UPS, Toulouse, France

12 LAL, CNRS/IN2P3, University Paris-Saclay, Orsay, France

13 MPA, Garching, Germany 
14 MPE, Garching, Germany

15 University Observatory Munich, Ludwig-Maximimilians University Munich, Munich, Germany

16 IISER-TVM, Thiruvananthapuram, India

17 National University of Ireland Maynooth, Maynooth, Ireland

18 ASI, Rome, Italy

19 IFPU, Grignano, Italy

20 INAF - OAS Bologna, Bologna, Italy

21 INFN Ferrara, Ferrara, Italy

22 INFN Milano, Milan, Italy

23 INFN Pisa, Pisa, Italy

24 INFN Roma, Rome, Italy

25 INFN Trieste, Trieste, Italy

26 Sapienza University of Rome, Rome, Italy

27 SISSA, Trieste, Italy

28 University di Ferrara, Ferrara, Italy

29 University di Roma Tor Vergata, Rome, Italy

30 University of Milano, Milan, Italy

31 University of Milano Bicocca, Milan, Italy

32 AIST, Tsukuba, Japan

33 Department of Astronomy, University of Tokyo, Tokyo, Japan

34 Department of Physics, University of Tokyo, Tokyo, Japan

35 GSFS, University of Tokyo, Kashiwa, Japan

IPST, University of Tokyo, Tokyo, Japan

37 ISAS, JAXA, Sagamihara, Japan

38 ISSP, University of Tokyo, Kashiwa, Japan

39 JAXA, Tsukuba, Japan

40 Kavli IPMU (WPI), University of Tokyo, Kashiwa, Japan

41 KEK, Tsukuba, Japan

42 Kitasato University, Sagamihara, Japan

43 Konan University, Kobe, Japan

44 Kurume University, Kurume, Japan

45 Nagoya University, Nagoya, Japan

46 NAOJ, Mitaka, Japan

47 National Institute of Technology, Kagawa College, Takamatsu, Japan

48 Okayama University, Okayama, Japan

49 Osaka Prefecture University, Sakai, Japan

50 RESCEU, University of Tokyo, Tokyo, Japan

51 Saitama University, Saitama, Japan 
52 SOKENDAI, Hayama, Japan

53 Tohoku University, Sendai, Japan

54 Waseda University, Tokyo, Japan

55 Yokohama National University, Yokohama, Japan

56 SRON, Utrecht, The Netherlands

57 University of Oslo, Oslo, Norway

58 IAC, Santa Cruz de Tenerife, Spain

59 IFCA, CSIC-Univ de Cantabria, Santander, Spain

60 University de La Laguna, Santa Cruz de Tenerife, Spain

61 Stockholm University, Stockholm, Sweden

62 Cardiff University, Cardiff, UK

63 KICC, Cambridge, UK

64 University College London, London, UK

65 University of Cambridge, Cambridge, UK

66 University of Manchester, Manchester, UK

67 University of Oxford, Oxford, UK

68 Cornell University, Ithaca, USA

69 KIPAC, Menlo Park, USA

70 LBNL, Berkeley, USA

71 NASA Goddard, Greenbelt, USA

72 NIST, Boulder, USA

73 Stanford University, Stanford, USA

74 University of California, Berkeley, USA

75 University of California, San Diego, USA

76 University of Colorado, Boulder, USA

77 USTH, Hanoi, Vietnam 